\title{
Factors Affecting the Mechanism of 1,3-Butadiene Polymerization at Open Metal Sites in Co-MFU-4l
}

\author{
Huiling Shao ${ }^{\dagger, *}$, Riddhish Pandharkar ${ }^{\dagger, t}$, Christopher J. Cramer ${ }^{\dagger, a}$ \\ shaoh@umn.edu \\ ${ }^{\dagger}$ Department of Chemistry, Minnesota Supercomputing Institute and Chemical Theory Center, \\ University of Minnesota, Minneapolis, Minnesota 55455, United States \\ ¥Department of Chemistry, Pritzker School of Molecular Engineering, James Franck Institute, Chicago Center for Theoretical \\ Chemistry, The University of Chicago, 5735 S Ellis Ave, Chicago, IL 60637, United States \\ ${ }^{a}$ Underwriters Laboratories Inc., 333 Pfingsten Rd., Northbrook, IL 60026
}

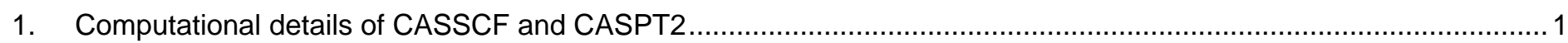

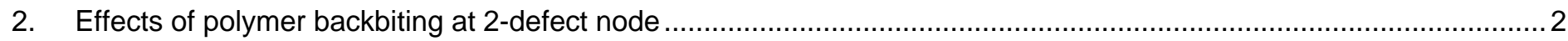

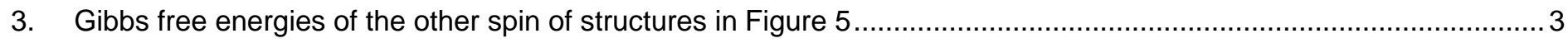

4. Initiation mechanism at pristine, singly defected, and doubly defected nodes ................................................. 4

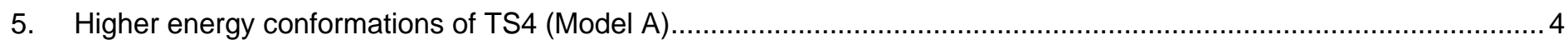

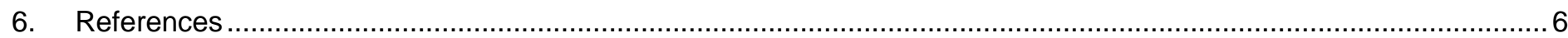

\section{Computational details of CASSCF and CASPT2}

An active space of the 11 electrons in 14 orbitals was chosen for CASSCF and CASPT2 calculations. The orbitals chosen include three occupied $3 \mathrm{~d}$ orbitals with their corresponding $4 \mathrm{~d}$ correlation orbitals and four bonding orbitals with their correlating pairs. Figure S1 presents the natural orbitals for the quartet state of IM1a. The orbitals for the other spin and intermediates are qualitatively similar to the ones shown in Figure S1.
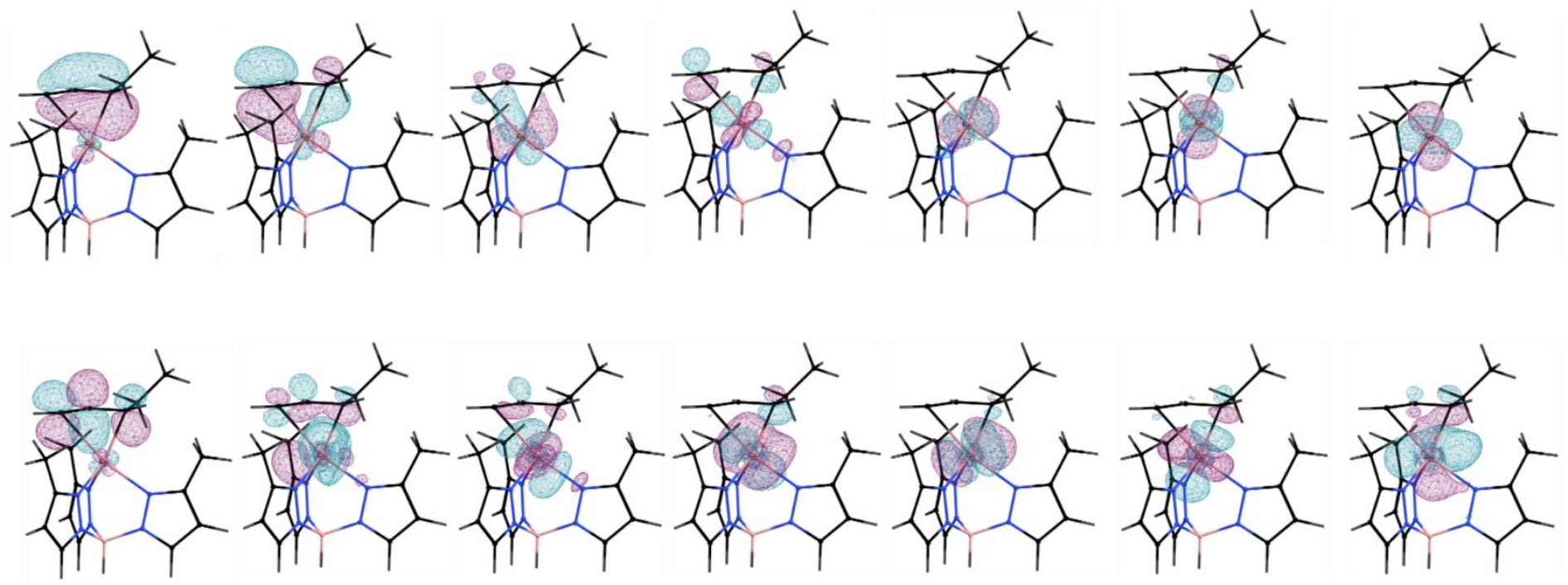

Figure S1: The active orbitals for the quartet state of IM1a. 
State specific calculations for both spin states were done separately at their respective geometries. The VTZP contraction of the ANO-RCC basis was used for the cobalt, while the VDZP contraction was used for the other elements. ${ }^{1}$ The DouglasKroll-Hess (DKH) Hamiltonian was used to include the scalar relativistic effects. ${ }^{2,3}$ Density fitting using a resolution of identity (RI) and Cholesky decomposition (CD) was used to make the calculations affordable. An imaginary shift of 0.2 was included in the energy denominators in CASPT2 to avoid singularities caused by intruder states. All multi-reference calculations were performed using OpenMolcas version $20.10 .^{4}$

Table S1. Computed double-quartet gaps with CASSCF and CASPT methods

\begin{tabular}{|c|c|c|}
\hline IM1A (pristine) & CASSCF (11,14) & CASPT2 (11,14) \\
\hline Doublet (Hartree) & -2402.48495 & -2406.48602 \\
\hline Quartet (Hartree) & -2402.48707 & -2406.45934 \\
\hline Gap (kcal/mol) & 1.3 & $\mathbf{- 1 6 . 7}$ \\
\hline IM1B (one-defect) & CASSCF (11,14) & -2142.654051 \\
\hline Doublet (Hartree) & -2139.56478 & -2142.62512 \\
\hline Quartet (Hartree) & -2139.55641 & $\mathbf{- 1 8 . 2}$ \\
\hline Gap (kcal/mol) & -5.3 & CASPT2 (11,14) \\
\hline IM1C (two-defects) & CASSCF (11,14) & -1878.80850 \\
\hline Doublet (Hartree) & -1876.62238 & -1878.78508 \\
\hline Quartet (Hartree) & -1876.61173 & $\mathbf{- 1 4 . 7}$ \\
\hline Gap (kcal/mol) & -6.7 & $\mathbf{1 4})$ \\
\hline
\end{tabular}

\section{Effects of polymer backbiting at 2-defect node}

We observed that polymer backbiting could effectively stabilize the under-coordinated Co(II) center at a doubly defected node (Figure S2). The presumably 3-coordinated Co-anti-allylic intermediate is stabilized by $2.7 \mathrm{kcal} / \mathrm{mol}$ (comparing IM1c with backbiting with IM-S1 without backbiting). Similarly, the presumably 2-coordinated anti-syn isomerization transition state is stabilized by $6.8 \mathrm{kcal} / \mathrm{mol}$ (comparing TS2c with backbiting with TS-S1 without backbiting). 


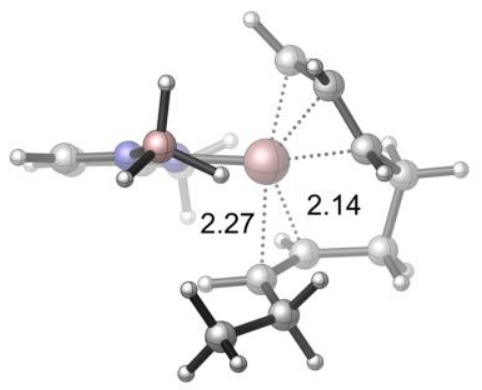

IM1c (with backbiting) $\Delta \mathrm{G}^{\ddagger}=0.0 \mathrm{kcal} / \mathrm{mol}$

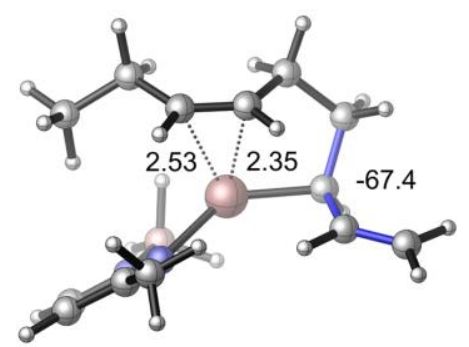

TS2c (with backbiting) $\Delta \mathrm{G}^{\ddagger}=30.7 \mathrm{kcal} / \mathrm{mol}$

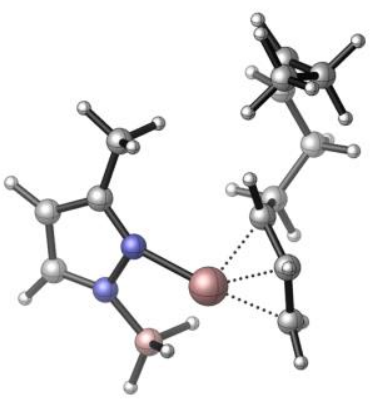

IM-S1 (no backbiting) $\Delta \mathrm{G}^{\ddagger}=2.7 \mathrm{kcal} / \mathrm{mol}$

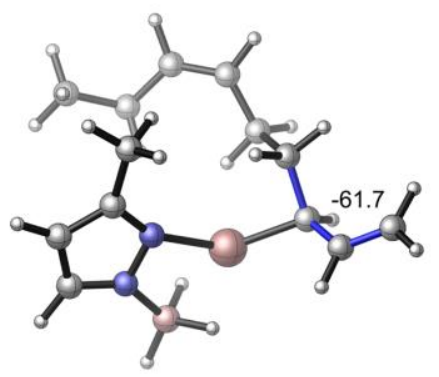

TS-S1 (no backbiting) $\Delta \mathrm{G}^{\ddagger}=37.5 \mathrm{kcal} / \mathrm{mol}$

Figure S2. Higher energy conformations of IM1c and TS2c without polymer backbiting

\section{Gibbs free energies of the other spin of structures in Figure 5}

We considered both doublet and quartet spin states for all intermediates and transition states by calculating the vertical single point energies (Table S2). We observed that the doublet state is more stable in IM1, IM2, IM3, IM4, TS1, and TS3. The quartet state is more stable in TS2.

Table S2. Computed Gibbs Free energies $(\mathrm{kcal} / \mathrm{mol})$ of both doublet and quartet spin states of all compounds in Figure 5

\begin{tabular}{|c|c|c|c|c|c|c|}
\hline \multirow{2}{*}{} & \multicolumn{2}{|c|}{ pristine node } & \multicolumn{2}{c|}{ 1 defect node } & \multicolumn{2}{c|}{ 2 defects node } \\
\cline { 2 - 7 } & doublet & quartet & doublet & quartet & doublet & quartet \\
\hline IM1 & 0.0 & 30.3 & 0.0 & 39.3 & 0.0 & 37.3 \\
\hline TS1 & 43.1 & 54.6 & 35.2 & 53.3 & 24.5 & 58.7 \\
\hline IM2 & -4.1 & 26.3 & -4.5 & 34.7 & -4.6 & 32.9 \\
\hline TS2 & 31.6 & 20.0 & 36.4 & 33.6 & 44.1 & 30.7 \\
\hline IM3 & -3.0 & 30.8 & -1.5 & 39.7 & -1.1 & 39.9 \\
\hline TS3 & 40.6 & 65.7 & 34.1 & 63.2 & 21.2 & 55.0 \\
\hline IM4 & -8.5 & 25.1 & -6.0 & 35.1 & -8.5 & 35.6 \\
\hline
\end{tabular}




\section{Initiation mechanism at pristine, singly defected, and doubly defected nodes}

We studied the initiation mechanism at pristine, singly defected, and doubly defected nodes (Table S3). Our computation results suggested that the cis-1-4-insertion (TS-S3) is favored over the other three competing insertion transition states at defected nodes ( $b$ and $c$ ). This observation agrees well with our main conclusion that 1,4-cis-PBD can only be selectively formed at defected nodes.

Table S3. Computed Gibbs Free energies of initiation transition states<smiles>[R]C(C=C)CO[Na]</smiles>

IM-S4

TS-S4 (trans-1-2 insertion)

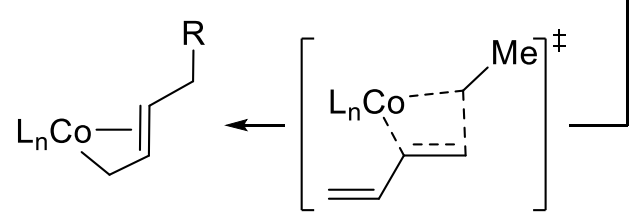

IM-S5
TS-S5

(trans-1-4 insertion)

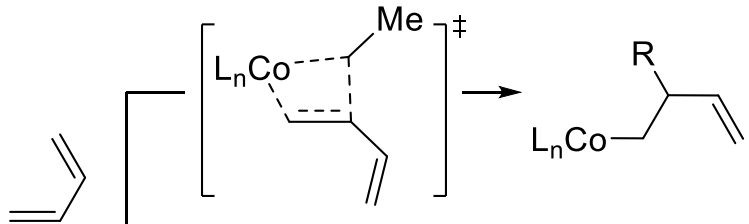

TS-S2

IM-S3 (cis-1-2 insertion)

IM-S2

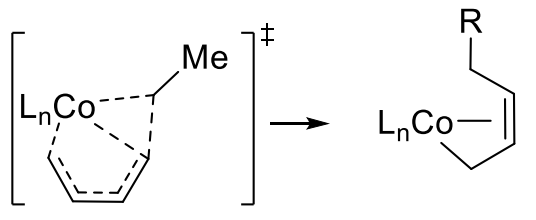

TS-S3

IM1

\begin{tabular}{|c|c|c|c|}
\hline$\Delta \mathrm{G}^{\ddagger}(\mathrm{kcal} / \mathrm{mol})$ & pristine node $(\mathrm{a})$ & 1 defect node $(\mathrm{b})$ & 2 defects node $(\mathrm{c})$ \\
\hline IM-S2 & 0.0 & 0.0 & 0.0. \\
\hline TS-S2 & 30.1 & 23.7 & 14.9 \\
\hline TS-S3 & 32.3 & 10.0 & 9.1 \\
\hline TS-S4 & 28.8 & 17.0 & 15.8 \\
\hline TS-S5 & 34.6 & 20.4 & 12.3 \\
\hline
\end{tabular}

\section{Higher energy conformations of TS4 (Model A)}

We carefully evaluated higher energy conformations of the stereoisomeric (TS4) transition states with model A (Figure

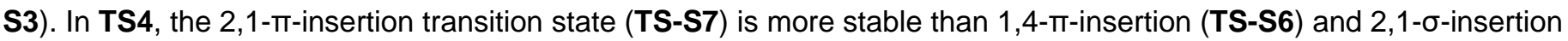
with polymer backbiting (TS-S8). In the main article, we then studied the origin of selectivity in the main article (TS4C, Figure 8) using the most stable 2,1-ח-insertion transition state. 


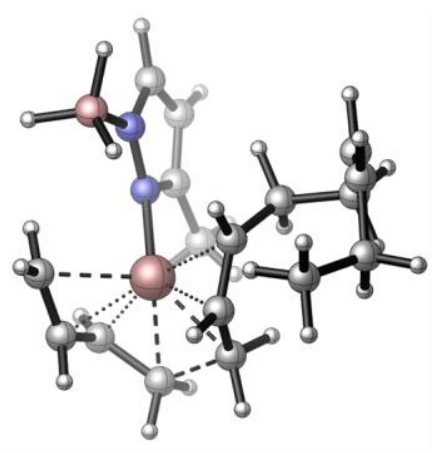

TS-S6

1,4-m-insertion

$(45.6)$

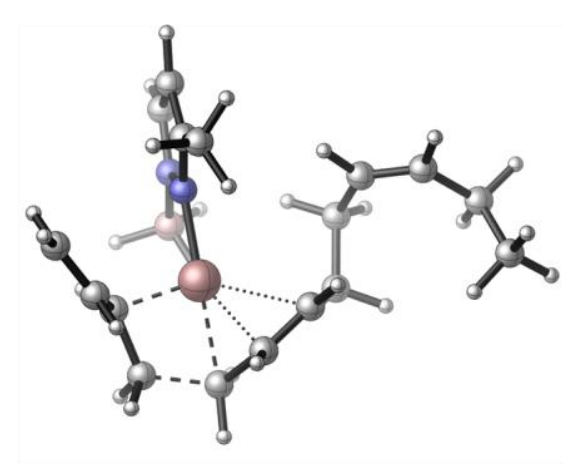

TS-S7

2,1-m-insertion

(31.8)

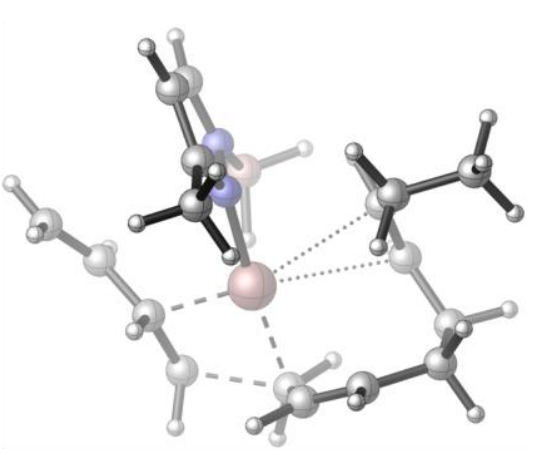

TS-S8

2,1- $\sigma$-insertion with backbiting

(44.1)

Figure S3. Higher energy conformations of TS4 


\section{References}

(1) Roos, B. O.; Lindh, R.; Malmqvist, P.-Å.; Veryazov, V.; Widmark, P.-O. Main group atoms and dimers studied with a new relativistic ANO basis set. J. Phys. Chem. A., 2004, 108, 2851-2858.

(2) Douglas, M.; Kroll, N. M. Quantum electrodynamical corrections to the fine structure of helium. Ann. Phys., 1974, 82, 89-155.

(3) Wolf, A.; Reiher, M.; Hess, B. A. The generalized Douglas--Kroll transformation. J. Chem. Phys., 2002, 117, 92159226.

(4) Fdez. Galván, I., Vacher, M., Alavi, A., Angeli, C., Aquilante, F., Autschbach, J., Bao, J.J., Bokarev, S.I., Bogdanov, N.A., Carlson, R.K. and Chibotaru, L.F., OpenMolcas: From source code to insight. J. Chem. Theory Comput., 2019. 15(11), 5925-5964. 